\title{
Light dosimetry in photodynamic therapy using optical fiber delivery
}

Steven L. Jacques, Martin R. Ostermeyer, Lihong V. Wang, Andreas H. Hielscher

Steven L. Jacques, Martin R. Ostermeyer, Lihong V. Wang, Andreas H. Hielscher, "Light dosimetry in photodynamic therapy using optical fiber delivery," Proc. SPIE 2133, Optical Methods for Tumor Treatment and Detection: Mechanisms and Techniques in Photodynamic Therapy III, (19 July 1994); doi: 10.1117/12.179977 


\title{
Light dosimetry in PDT using optical fiber delivery.
}

\author{
Steven L. Jacques, Martin Ostermeyer, Lihong Wang, Andreas Hielscher \\ Laser Biology Research Lab. \\ Univ. of Texas M. D. Anderson Cancer Center, Houston, TX 77030
}

\begin{abstract}
The paper considers rapid calculations of light distributions in a tissue for photodynamic therapy. The delivery system considered is a single optical fiber, although other configurations are easily modeled. The influence of optical heterogeneities in the tissue is considered.

The optical distributions surrounding an optical fiber within a tissue can be well approximated by a point source of light placed one "reduced mean free path" ( $\left.m f p^{\prime}\right)$ in front of the fiber tip. One mfp' equals $1 /\left(\mu_{\mathrm{a}}+\mu_{\mathrm{s}}(1-\mathrm{g})\right)$. Then the simple diffusion theory expression for $3 \mathrm{D}$ diffusion from a point source predicts the light distribution in the tissue.

The influence of optical heterogeneities in the tissue can also be well approximated. A method is described for representing regions of increased absorption (such as a region with increased vascularity) as virtual sources of "negative radiant power" such that linear superposition of the true primary source and the virtual sources yields the net light distribution in the heterogeneous tissue. The method is rapid, flexible, and generally accurate to within about $15 \%$ error. The method yields light distributions in optically complex tissues.
\end{abstract}

\section{INTRODUCTION}

During photodynamic therapy, the ability of light to penetrate a tissue is limited by absorbing and scattering properties of the tissue. A tumor often will have optical properties not too dissimilar from the surrounding normal tissue, but the presence of increased tumor vascularity may increase the local average absorption coefficient. Also, as PDT proceeds, the effect of PDT on the tumor vasculature in some cases has been reported to be an increase in the density of blood in the tumor which further decreases the light penetration.

This paper addresses two issues: (1) What is the light distribution around an optical fiber imbedded within a homogeneous tissue? (2) How can one calculate the effect of optical heterogeneities caused by regions of increased light absorption (such as increased blood content)?

\section{THEORY}

\subsection{An optical fiber within a homogeneous tissue}

When an imbedded optical fiber delivers light to a homogeneous tissue, the light distribution (fluence rate, $\mathrm{F},\left[\mathrm{W} / \mathrm{cm}^{2}\right]$ ) can be well predicted in regions distant from the source by the simple diffusion theory expression. If the source has a power of $1 \mathrm{~W}$, the fluence rate is: 


$$
\mathrm{F}(\mathrm{r})=\frac{1}{4 \pi \mu_{\mathrm{a}} \delta^{2}} \frac{\exp (-\mathrm{r} / \delta)}{\mathrm{r}}\left[\mathrm{W} / \mathrm{cm}^{2}\right]
$$

where $\delta$ is the optical penetration depth:

$$
\delta=\frac{1}{\sqrt{3 \mu_{\mathrm{a}}\left(\mu_{\mathrm{a}}+\mu_{\mathrm{s}}(1-\mathrm{g})\right)}}[\mathrm{cm}]
$$

The light appears to diffuse from a central source point $(r=0)$ which is located just in front of the optical fiber (see Fig. 1). The distance between the tip of the fiber and the apparent center of the spherical diffusion field is one "reduced mean free path" (mfp'):

$$
\mathrm{mfp}^{\prime}=\frac{1}{\mu_{\mathrm{a}}+\mu_{\mathrm{s}}(1-\mathrm{g})}
$$

This data for this conclusion is not shown here, but is the result of our computer simulations and experiments.

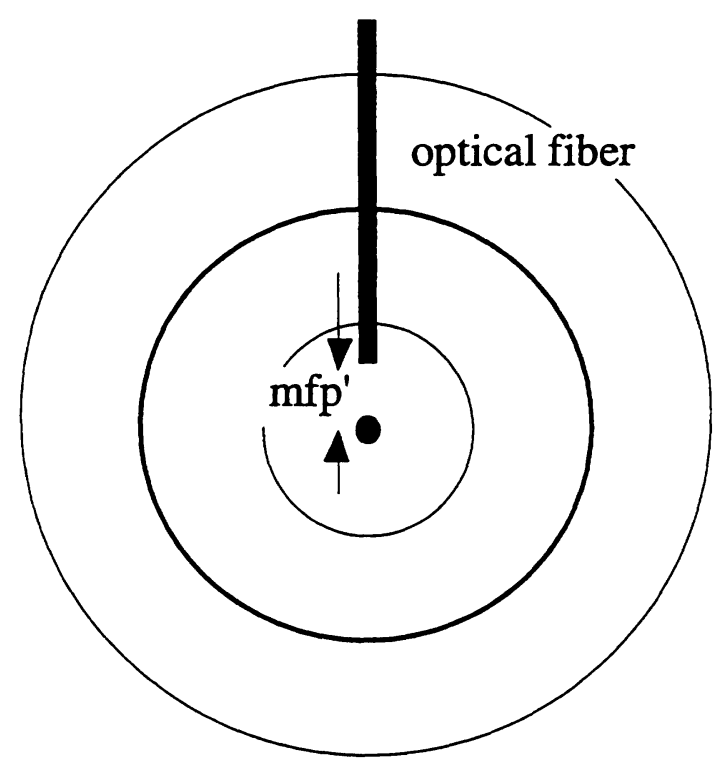

Fig. 1: When an optical fiber delivers light, a wave of spherical light diffusion is generated whose center is located $1 \mathrm{mfp}$ ' in front of the tip of an optical fiber.

\subsection{A single small absorbing object within a homogeneous tissue}

To treat the case of regions of increased absorbance within an otherwise homogeneous tissue, a simple method of calculation was devised. Assume there is a single point source of light $\left(\mathrm{S}_{0}[\mathrm{~W}]\right)$ located at the origin of our coordinate system.

Consider a very small volume $\left(V_{1}\right)$ of tissue which has an absorption coefficient $\left(\mu_{\mathrm{a} 1}\right)$ which is elevated relative to the surrounding tissue with homogeneous optical properties $\left(\mu_{\mathrm{a}}, \mu_{\mathrm{s}}, \mathrm{g}\right)$. The center of the small volume is at a distance $r_{1}$ from the point source at the origin. The amount of light incident on that volume is specified: 


$$
F\left(r_{1}\right)=S_{0} C\left(r_{1}\right)=S_{0} \frac{1}{4 \pi \mu_{a} \delta^{2}} \frac{\exp \left(-r_{1} / \delta\right)}{r_{1}}
$$

where $C\left(r_{1}\right)$ refers to the coupling factor $\left[\mathrm{cm}^{-2}\right]$ between the source $S_{0}[W]$ and the fluence $\left[\mathrm{W} / \mathrm{cm}^{2}\right]$ at position $\mathrm{r}_{1}$, and is identical to Eq. 1 . The amount of light additionally absorbed by the small volume, relative to the background absorption, is well approximated by the calculation:

$$
\mathrm{Q}_{1}=\left(\mu_{\mathrm{a} 1}-\mu_{\mathrm{a}}\right) \mathrm{F}\left(\mathrm{r}_{1}\right) \mathrm{V}_{1} \quad[\mathrm{~W}]
$$

The influence of this locally absorbed power $\mathrm{Q}_{1}$ on the overall light field can be approximated by assigning a source value, $S_{1}=-Q_{1}$, to a virtual point source of light centered on the small volume, negative in sign, which superimposes a negative field of fluence rate onto the original field due to the primary source at the origin.

Therefore, the net field of light at any position $r$, or $(x, y, z)$, is described:

$$
F(r)=\frac{S_{0}}{4 \pi \mu_{a} \delta^{2}} \frac{\exp (-r / \delta)}{r}+\frac{S_{1}}{4 \pi \mu_{a} \delta^{2}} \frac{\exp \left(-r^{*} / \delta\right)}{r^{*}}
$$

where $r$ is the distance from the point source at the origin to some observation point $(x, y, z)$ :

$$
r=\sqrt{x^{2}+y^{2}+z^{2}}
$$

and $r^{*}$ refers to the distance between the observation point $(x, y, z)$ and the position of the small volume $\left(\mathrm{x}_{1}, \mathrm{y}_{1}, \mathrm{z}_{1}\right)$ :

$$
r^{*}=\sqrt{\left(x-x_{1}\right)^{2}+\left(y-y_{1}\right)^{2}+\left(z-z_{1}\right)^{2}}
$$

and the term $S_{1}$ has a negative value:

$$
\mathrm{S}_{1}=-\left(\mu_{\mathrm{a} 1}-\mu_{\mathrm{a}}\right) \mathrm{F}(\mathrm{n}) \mathrm{V}_{1}
$$

The above example illustrates the case of a single small object with only a slightly elevated absorption coefficient relative to that of the surrounding tissue. Therefore, the small object only slightly perturbs the light field. The fluence rate at the center of the volume is approximately equal to the mean of the fluence at the front and rear of the volume along the axis defined by the origin and the position of the volume.

\subsection{Multiple absorbing objects}

If there are several small absorbing objects within the tissue, their influence can still be calculated using superposition. However, one must allow for the mutual influence of the objects on each other. With many objects, some objects will shade other objects from the primary source. Despite the interaction of the many objects, the problem remains a linear system which can be solved by standard matrix operations. In this section, we first present the solution as an iterative matrix calculation to illustrate the nature of the solution. In the next section, we restate the solution as solvable by standard matrix operations. 
Let us continue to assume a single point source at the origin, although this is not in general a necessary restriction. The primary source is $\mathrm{S}_{0}[\mathrm{~W}]$. Consider $\mathrm{N}$ small absorbing objects in the tissue. Each object can act as a virtual source once the power of absorption by each object is specified. Hence, the various sources, $S_{0}$ and $S_{1}$ to $S_{N}$, can be expressed as a source vector $\mathbf{S}$.

The coupling between the primary source $S_{0}$ and each object can be calculated by Eq. 1. Also, the coupling between each virtual source $S_{i}$ and the other $N-1$ virtual sources can also be calculated. The general form of the coupling coefficient, $\mathrm{C}_{\mathrm{ij}}$, is:

$$
\mathrm{C}_{\mathrm{ij}}=\frac{1}{4 \pi \mu_{\mathrm{a}} \delta^{2}} \frac{\exp \left(-\mathrm{r}_{\mathrm{ij}} / \delta\right)}{\mathrm{r}_{\mathrm{ij}}} \quad\left[\mathrm{cm}^{-2}\right]
$$

where the distance between the $\mathrm{i}^{\text {th }}$ and $\mathrm{j}^{\text {th }}$ object is $\mathrm{r}_{\mathrm{ij}}$ :

$$
r_{i j}=\sqrt{\left(x_{i}-x_{j}\right)^{2}+\left(y_{i}-y_{j}\right)^{2}+\left(z_{i}-z_{j}\right)^{2}}
$$

The collection of all coupling coefficients constitutes a coupling matrix $\mathbf{C}$.

For the special case of $j=i$, the value $r_{i i}$ is zero which causes trouble in the denominator of Eq. 10. In this case, the value of $C_{i i}$ is calculated by the following expression based on a uniform distribution of energy deposition within the small (spherical) absorbing object whose radius is R:

$$
\mathrm{C}_{\mathrm{ii}}=\frac{3}{4 \pi \mathrm{R}^{3} \mu_{\mathrm{a}}}(1-(\mathrm{R} / \delta+1) \exp (-\mathrm{R} / \delta)) \quad\left[\mathrm{cm}^{-2}\right]
$$

The fluence rates $F_{i}$ at each $i^{\text {th }}$ object can be calculated by the sum:

$$
F_{i}=\sum_{j=0}^{N} C_{i j} S_{j}
$$

The set of fluence rates $F_{i}$ can be expressed as a fluence rate vector $F$.

Combining the fluence rate vector $\mathbf{F}$, the coupling matrix $\mathbf{C}$, and the source vector $\mathbf{S}$ into a single expression yields:

$$
\begin{gathered}
\mathbf{F}=\mathbf{C S} \\
\text { or } \\
{\left[\begin{array}{c}
F_{1} \\
F_{2} \\
F_{3} \\
\cdot \\
\cdot \\
F_{N}
\end{array}\right]=\left[\begin{array}{cccccc}
C_{10} & C_{11} & C_{12} & \cdot & \cdot & C_{1 N} \\
C_{20} & C_{21} & C_{22} & \cdot & \cdot & C_{2 N} \\
C_{30} & C_{31} & C_{32} & \cdot & \cdot & C_{3 N} \\
\cdot & \cdot & \cdot & \cdot & \cdot & \cdot \\
\cdot & \cdot & \cdot & \cdot & \cdot & \cdot \\
C_{N} & C_{N 1} & C_{N 2} & \cdot & \cdot & C_{N N} \\
S_{0} \\
S_{1} \\
S_{2} \\
S_{3} \\
\cdot \\
S_{N}
\end{array}\right]}
\end{gathered}
$$


Initially, let the sources $S_{1}$ to $S_{N}$ equal zero. The first calculation of $\mathbf{F}$ is:

$$
\mathrm{F}_{\mathrm{i}}=\mathrm{C}_{\mathrm{i} 0} \mathrm{~S}_{0}
$$

Then the $\mathbf{S}$ vector is updated based on the new $\mathbf{F}$ :

$$
S_{i}=-\left(\mu_{a i}-\mu_{a}\right) F_{i} V_{i} \text { for } i=1 \text { to } N
$$

Now, each object has a negative virtual source value $S_{i}$. The matrix multiplication is repeated to yield a new $\mathbf{F}$ vector which now includes some negative influence imposed by all the virtual sources. In other words, the interaction between all virtual sources is affecting the fluence rate at each object. Accounting for such interaction properly accounts for the shading of some objects by other objects.

Again, the $\mathbf{S}$ vector is recalculated. This cycle of calculating $\mathbf{F}$ then $\mathbf{S}$ is iterated until the values for $\mathbf{S}$ stabilize to within some convergence criteria. The iteration is described by the algorithm:

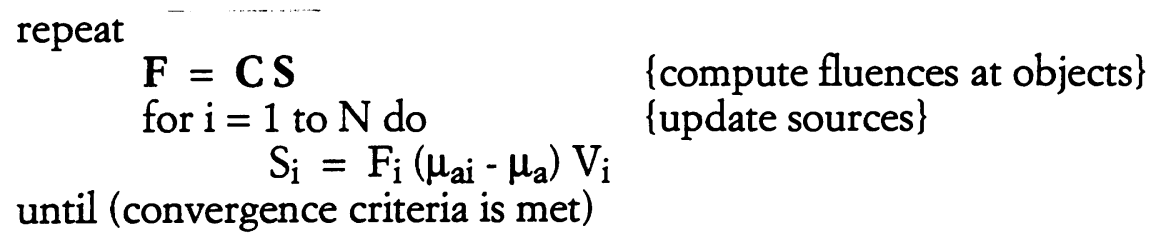

Once $\mathbf{S}$ has converged, the fluence rate at any arbitrary point $r$, or $(x, y, z)$, can be calculated:

where

$$
F(r)=\sum_{i=0}^{N} S_{i} \frac{\exp \left(-r_{i} / \delta\right)}{4 \pi \mu_{a} \delta^{2} r_{i}}
$$

$$
\mathrm{r}_{\mathrm{i}}=\sqrt{\left(\mathrm{x}-\mathrm{x}_{\mathrm{i}}\right)^{2}+\left(\mathrm{y}-\mathrm{y}_{\mathrm{i}}\right)^{2}+\left(\mathrm{z}-\mathrm{z}_{\mathrm{i}}\right)^{2}}
$$

\subsection{Alternative standard matrix solution}

The above section describes the solution by iterative matrix operations in order to better illustrate to some readers how the various objects interact. But a more concise description is given by the matrix identity relation:

$$
\begin{aligned}
& \mathbf{S}=\mathbf{M S} \\
& \text { or } \\
& {\left[\begin{array}{c}
S_{0} \\
S_{1} \\
S_{2} \\
\cdot \\
S_{N}
\end{array}\right]=\left[\begin{array}{cccccc}
1 & 0 & 0 & \cdot & \cdot & 0 \\
M_{10} & M_{11} & M_{12} & \cdot & \cdot & M_{1 N} \\
M_{20} & M_{21} & M_{22} & \cdot & \cdot & M_{2 N} \\
M_{30} & M_{31} & M_{32} & \cdot & \cdot & M_{3 N} \\
\cdot & \cdot & \cdot & \cdot & \cdot & S_{1} \\
\mathrm{M}_{N} & M_{N 1} & M_{N 2} & \cdot & \cdot & M_{N N} \\
\cdot \\
\cdot
\end{array}\right]}
\end{aligned}
$$


where

and

$$
M_{i j}=-\left(\mu_{a i}-\mu_{a}\right) V_{i} \frac{\exp \left(-r_{i j} / \delta\right)}{4 \pi \mu_{a} \delta^{2} r_{i j}}
$$

$$
r_{i j}=\sqrt{\left(x_{i}-x_{j}\right)^{2}+\left(y_{i}-y_{j}\right)^{2}+\left(z_{i}-z_{j}\right)^{2}}
$$

The solution of Eq. 20 involves standard linear matrix operations to transform $\mathbf{M}$ into an identity matrix. The operations also transform the $\mathbf{S}$ vector which yields the solution for $\mathbf{S}$. ( $\mathrm{S}_{0}$ is positive and $S_{i}$ for $i>0$ is negative if $\mu_{\mathrm{ai}}>\mu_{\mathrm{a}}$ and positive if $\mu_{\mathrm{ai}}<\mu_{\mathrm{a}}$ ).

\section{AN EXAMPLE}

To illustrate the use of the above method, we considered the light distribution in a tissue from a single optical fiber with two spherical tumors that presented regions of increased absorption (Fig. 2). The background optical properties of the tissue were $\left(\mu_{\mathrm{a}}, \mu_{\mathrm{s}}, \mathrm{g}\right)$ equal $\left(0.1 \mathrm{~cm}^{-1}, 120 \mathrm{~cm}^{-1}, 0.9\right)$ which are typical for many tissues in the red region $(\sim 630 \mathrm{~nm})$ of the visible spectrum. The optical penetration depth, $\delta$, equaled $0.52 \mathrm{~cm}$. The two spherical tumors had the same scattering properties as the background tissue, but the absorption coefficient was increased to $1 \mathrm{~cm}^{-1}$ due to increased vascularity. These choices do not represent all or most tumors, but were simply for illustration.

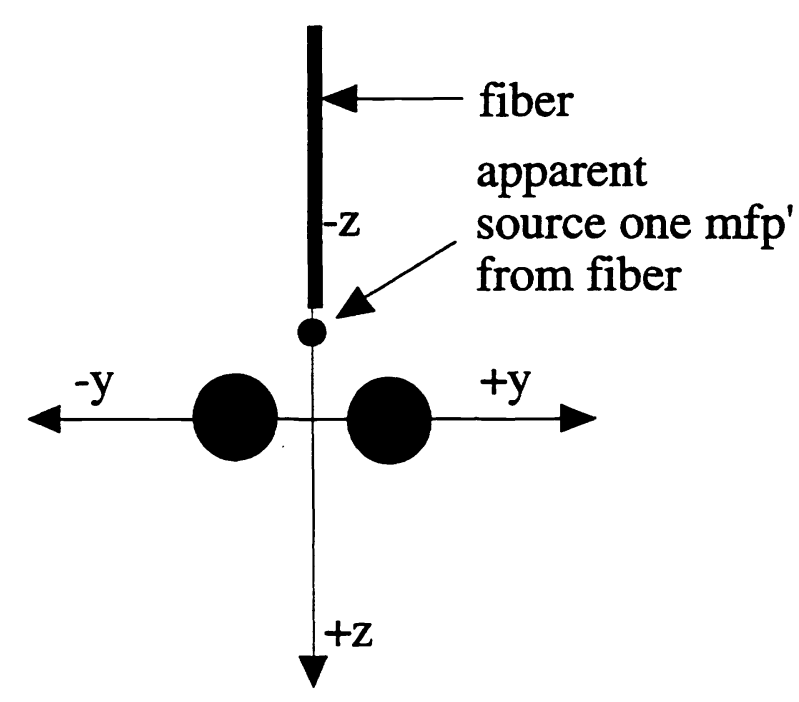

Fig. 2: Geometry of example problem: two spherical tumors and one optical fiber.

The primary source was an isotropic point source located one $\mathrm{mfp}$ ( or $0.083 \mathrm{~cm}$ ) from the tip of the fiber; i.e., the source was at $(x, y, z)$ equal $(0,0,-0.38 \mathrm{~cm})$. The two spheres had a diameter of $0.2 \mathrm{~cm}$ and were located at $(0,+Y \mathrm{~cm}, 0)$ and $(0,-Y \mathrm{~cm}, 0)$, where the positions $\pm Y$ were varied.

The two $0.2-\mathrm{cm}$ diameter spheres were subdivided into 57 closest-packed spherical objects (Fig. 3). Consequently, the problem involved one source and (2)(57) or 114 objects. Each object had a radius such that its volume $(\mathrm{V})$ constituted $1 / 57$ th of the original spherical tumor volume $\left(\mathrm{V}_{\mathrm{t}}\right)$. Each object presented a sufficiently small optical depth such that it met the criterion that it only slightly perturbed the background light field. 


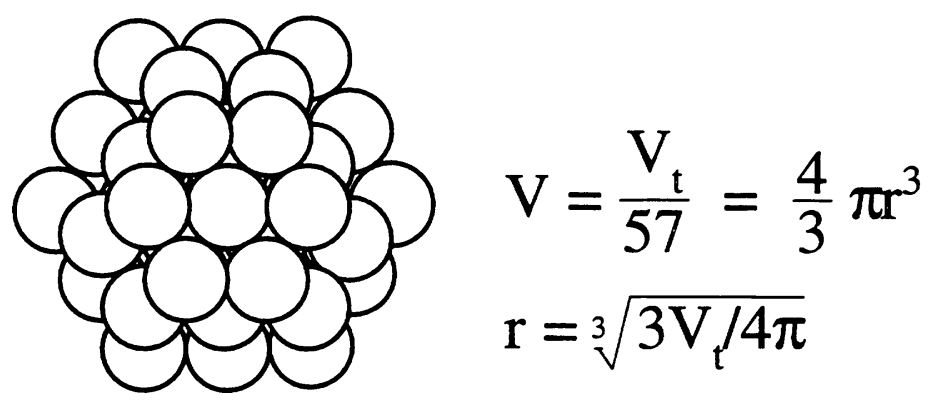

Fig. 3: Each spherical tumor was subdivided into 57 closest-packed spherical objects. This subdivision allowed the solution to account for how the front portion of each tumor closest to the point source shaded the rear portion of each tumor.

The problem was solved with the two spherical tumors located either $0.4 \mathrm{~cm}$ or $0.8 \mathrm{~cm}$ apart.

Solving the problem involved the following steps. First, the positions of each of the 114 objects were established. Then the coupling coefficients, $\mathrm{C}_{\mathrm{ij}}$, were calculated. Then the iterative matrix multiplication was executed until the sum of all $S_{i}$ had converged to within 1 part in a $10^{5}$, which took only 9 iterations. Finally, the fluence rate at a 30x30 grid of positions were calculated and compared with the the fluence rate in the absence of the objects to yield the relative fluence rate:

$$
\text { relative } F=\frac{F_{+o b j}}{F_{-o b j}}=\frac{\text { fluence rate with object }}{\text { fluence rate without object }}
$$

The iterative matrix calculation was very rapid. The program was written in standard ANSI C and run on a Sun workstation. The program execution, including storage of results on a hard disk, required only about $8 \mathrm{~s}$.

The spatial distribution of the relative $F$ is shown in the Figs. 5 and 6 for the $0.4-\mathrm{cm}$ and 0.8$\mathrm{cm}$ spacings between the tumors. The perspective of these figures is first illustrated in Fig. 4.

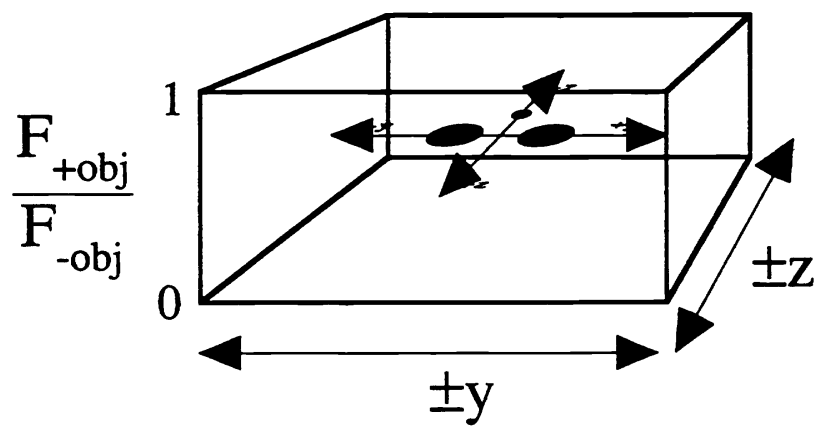

Fig. 4: Perspective used for Figs. 5 and 6 for the example problem shown in Fig. 2. The relative fluence rate, $F_{+o b j} / F_{-o b j}$, shows the effect of the two spherical tumors on the background field of light. 
Fig. 5: The relative fluence rate, $F_{+o b j} / F_{-o b j}$, for the example depicted in Fig. 2, using the perspective shown in Fig. 4 . The two $0.2-\mathrm{cm}$-diameter spherical tumors were placed $0.8 \mathrm{~cm}$ apart. The optical penetration depth, $\delta$, for the background tissue was $0.52 \mathrm{~cm}$. Contour lines have equal value spacings.
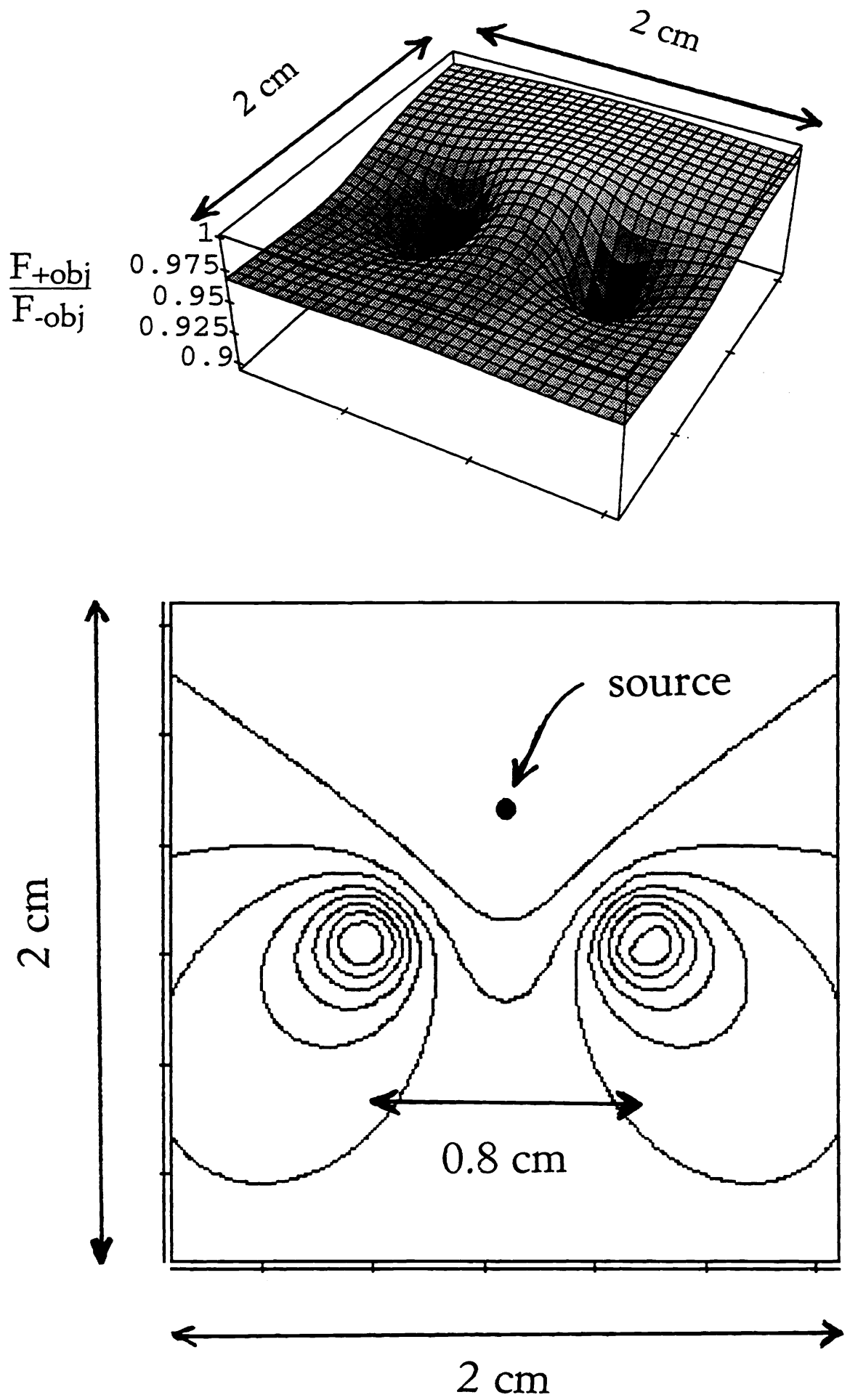
Fig. 6: The relative fluence rate, $\mathrm{F}_{+\mathrm{obj}} / \mathrm{F}_{-\mathrm{obj}}$, for the example depicted in Fig. 2, using the perspective shown in Fig. 4. The two $0.2-\mathrm{cm}$-diameter spherical tumors were placed $0.4 \mathrm{~cm}$ apart. The optical penetration depth, $\delta$, for the background tissue was $0.52 \mathrm{~cm}$. Contour lines have equal value spacings.
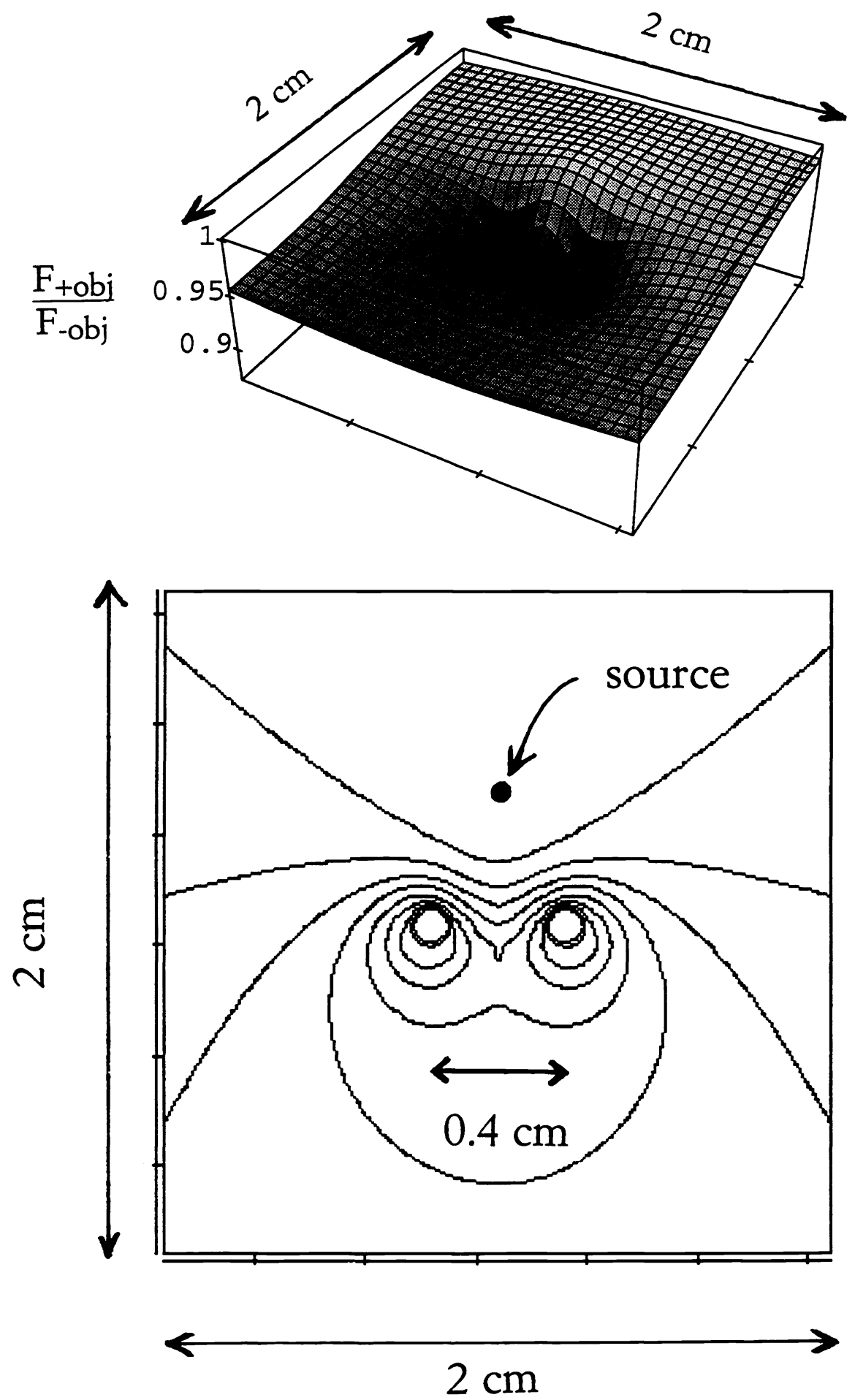


\section{CONCLUSION}

(1) Light dosimetry from an optical fiber can be well approximated by a point source placed one $\mathrm{mfp}$ ' from the tip of the fiber. (This conclusion is based on computer simulations and experiments not presented in this paper.)

(2) The influence of absorbing structures within the tissue, such as a tumor with elevated vascularity, can be approximated by subdividing large objects into smaller objects then treating the power additionally absorbed (above the background absorption) by each small object as a virtual source of negative power. Linear superposition of the primary and virtual sources using matrix calculations yields the values of the virtual sources which properly accounts for the shading of some objects by others. Once the virtual sources are specified, simple linear superposition of the contributions from the primary and virtual sources to any point in the field can be calculated.

Although this paper does not present information on the accuracy of this method, work in progress shows that accuracy is generally within about $15 \%$ relative to more accurate (but slow) calculation methods.

\section{ACKNOWLEDGEMENTS}

This work was supported by the Dept. of Energy (DE-FG05-91ER617226) and the NIH (R29-HL45045). 